\title{
Efeitos do exercício físico no controle clínico da asma
}

\author{
Effects of exercise on clinical asthma control
}

\section{Patrícia Duarte Freitas ${ }^{1}$, Ronaldo Aparecido da Silva ${ }^{2}$, Celso Ricardo Fernandes de Carvalho ${ }^{3}$}

Freitas PD, Silva RA, Carvalho CRF. Efeitos do exercício físico no controle clínico da asma / Effects of exercise on clinical asthma control. Rev Med (São Paulo). 2015 set.-dez.;94(4):246-55.

RESUMO: A asma é uma doença inflamatória crônica das vias aéreas cujo diagnóstico é clínico e o tratamento visa principalmente controlar os sintomas e diminuir os riscos de exacerbação. Mesmo sob tratamento clínico-medicamentoso adequado, os pacientes nem sempre atingem o controle clínico adequado. Por isso, recomenda-se o uso de terapias não farmacológicas, destacando-se o exercício físico (EF), que é, atualmente, reconhecido como parte fundamental do programa de reabilitação para asmáticos. A revisão que se segue objetivou explorar os aspectos relacionados à melhora do controle clínico da doença induzidos pelo EF em pacientes asmáticos. Para tanto, foram revisados artigos publicados na base de dados Pubmed e SciELO (de 1970 a 2015). Verificou-se que os primeiros estudos sugeririam que o EF, predominantemente aeróbio, melhora o condicionamento físico e a percepção de falta de ar (dispneia). Essa tendência foi mantida até os anos 90 e a partir de então surgiram estudos com maior rigor metodológico mostrando que o $\mathrm{EF}$ pode reduzir o broncoespasmo induzido pelo exercício (BIE), a responsividade brônquica bem como e a capacidade física aeróbia. Evidências mostrando que o EF melhora os fatores de saúde relacionados à qualidade de vida, o controle clínico da asma e a inflamação pulmonar passaram a ser melhor investigados apenas nos últimos 5 anos. Atualmente, considera-se a prática de EF como um componente fundamental no programa de tratamento para pacientes com asma moderada e grave, quando realizado com predominância aeróbia, em intensidade de baixa a moderada e feito pelo menos duas vezes por semana de forma individualizada. Portanto, o exercício físico parece potencializar o controle clínico da asma através da melhora do condicionamento físico, sendo importante sua indicação na prática clínica para os pacientes que estão em tratamento clínico-medicamentoso otimizado.

Descritores: Asma; Exercício; Controle; sinais e sintomas; Dispneia.

\begin{abstract}
Asthma is a chronic inflammatory airway disease whose diagnosis is clinical and treatment is aimed mainly control the symptoms and reduce the risk of exacerbation. Even under appropriate clinical and drug treatment, patients do not always reach the appropriate clinical management. Therefore, it is recommended to use of non-pharmacological therapies, highlighting the physical exercise (PE), which is currently recognized as a fundamental part of the rehabilitation program for asthmatics. The following review aimed to explore aspects related to improved clinical management of the disease induced by PE in patients with asthma. To this end, we reviewed articles published in the database Pubmed and SciELO (from 1970 to 2015). It was found that early studies suggest that PE, predominately aerobic, improves physical and perception of breathlessness (dyspnea). This trend was maintained until the $90 \mathrm{~s}$ and thereafter emerged studies with greater methodological rigor showing that PE can reduce exercise-induced bronchospasm (EIB), bronchial responsiveness and the aerobic capacity. Evidence showing that the PE improves health factors related to quality of life, clinical control of asthma and lung inflammation have become better investigated only in the last 5 years. Currently, it is considered the PE practice as a fundamental component in the treatment program for patients with moderate and severe asthma when performed predominantly aerobic, in low to moderate intensity and produced at least twice a week individually. Therefore, the physical exercise appears to enhance the clinical control of asthma by improving fitness, it is important indications in clinical practice for patients who are on optimal medical-drug treatment.
\end{abstract}

Keywords: Asthma; Exercise; Control; Signs and symptoms; Dyspnea.

Os autores colaboram igualmente para a realização desse artigo.

1. MSc, doutoranda, Departamento de Fisioterapia Fonoaudiologia e Terapia Ocupacional, Faculdade de Medicina, Universidade de São Paulo.

2. $\mathrm{PhD}$, pós-doutorando, Departamento de Fisioterapia Fonoaudiologia e Terapia Ocupacional, Faculdade de Medicina, Universidade de São Paulo. e-mail: ronaldo.experimental@gmail.com

3. Professor Livre docente, Departamento de Fisioterapia Fonoaudiologia e Terapia Ocupacional, Faculdade de Medicina, Universidade de São Paulo.

Endereço para correspondência: Celso Ricardo Fernandes de Carvalho. Faculdade de Medicina, Universidade de São Paulo. Av. Dr. Arnaldo, 455 - Cerqueira César - Sala 1210, $1^{\circ}$ andar, São Paulo, SP, Brasil. CEP: 01246-903. E-mail: cscarval@usp.br. 


\section{ASMA: DEFINIÇÃO, DIAGNÓSTICO E CONTROLE CLÍNICO}

A asma é uma doença crônica das vias aéreas caracterizada por limitação ao fluxo aéreo associado a sintomas respiratórios como sibilância, dispneia, aperto no peito e tosse que variam ao longo do tempo, quanto à sua ocorrência, frequência e intensidade ${ }^{1}$. Essas variações são, frequentemente, desencadeadas por fatores como exercício, exposição a alérgenos e/ou irritantes, mudanças no tempo ou infecções respiratórias virais. A asma está geralmente associada a uma inflamação crônica das vias aéreas e a hiperresponsividade brônquica, que podem persistirem mesmo quando os sintomas estão ausentes ou quando a função pulmonar está normal, podendo normalizar com o tratamento ${ }^{1}$. A doença possui elevada prevalência e morbidade, afetando de 1 a $18 \%$ da população em alguns países e no Brasil atinge, aproximadamente, $11 \%$ da população ${ }^{2}$.

O diagnóstico da asma é clínico e incluem presença e variação de sintomas respiratórios, histórico médico detalhado do paciente, confirmação da limitação ao fluxo aéreo expiratório através do teste de função pulmonar e reversibilidade positiva ao broncodilatador; podendo ser complementado por outros exames como variabilidade diurna diária do fluxo expiratório máximo, teste de broncoprovocação brônquica, dentre outros ${ }^{1}$. Após o diagnóstico, o controle clínico do paciente precisa ser monitorado periodicamente pela frequência de sintomas respiratórios (dias/semana), despertares noturnos relacionados à asma ou à limitação de atividades e frequência do uso de medicação de resgaste para alívio dos sintomas. Alguns instrumentos já foram desenvolvidos para avaliar e acompanhar o controle clínico através de pontos de corte que distinguem 3 níveis de controle dos sintomas (1), dentre eles se destacam os questionários "Asthma Control Questionnaire" (ACQ) e o "Asthma Control Test” (ACT), ambos já validados para o português ${ }^{3,4}$.

O ACQ possui 7 questões nas quais os pacientes são questionados a relembrar suas experiências na última semana. Seis questões se referem ao acordar à noite, sintomas ao acordar, limitação para as atividades, falta de ar, chiado e uso de beta 2 de curta duração e a última questão é obtida pelo valor percentual do volume expiratório forçado no primeiro segundo $\left(\mathrm{VEF}_{1}\right)$ obtido pré-broncodilatador ${ }^{5}$. Considera-se que valores $\geq 1,50$ indicam asma não-controlada, entre 0,75 e 1,5 parcialmente controlada e $<0,75$ totalmente controlado ${ }^{5,6}$ e variação de 0,5 ponto é considerada como melhora-ou-piora clinicamente significativas. Já o ACT possui 5 questões referentes aos sintomas, uso de medicação de alívio e efeito da asma nas atividades diárias avaliado nas últimas 4 semanas e classifica a asma em: totalmente descontrolada $<10$ pontos, asma parcialmente controlada entre 11 e 19 pontos e asma totalmente controlada $\geq 20$ pontos $^{7}$.

O tratamento da asma deve ser iniciado imediatamente após a confirmação do diagnóstico da doença objetivando controlar os sintomas e diminuir os riscos de exacerbação. O tratamento inclui a prescrição inicial da medicação (Figura 1), tratamento dos fatores de risco modificáveis, e terapias e estratégias não farmacológicas, destacando-se a prática de exercício físico. Os pacientes devem também participar de um programa de educação para asma, abrangendo informações sobre a fisiopatologia da doença, detectar e evitar os fatores gatilho, proficiência no uso do inalador, adesão, plano de ação escrito para a asma, automonitorização além de revisão médica regular ${ }^{1}$.

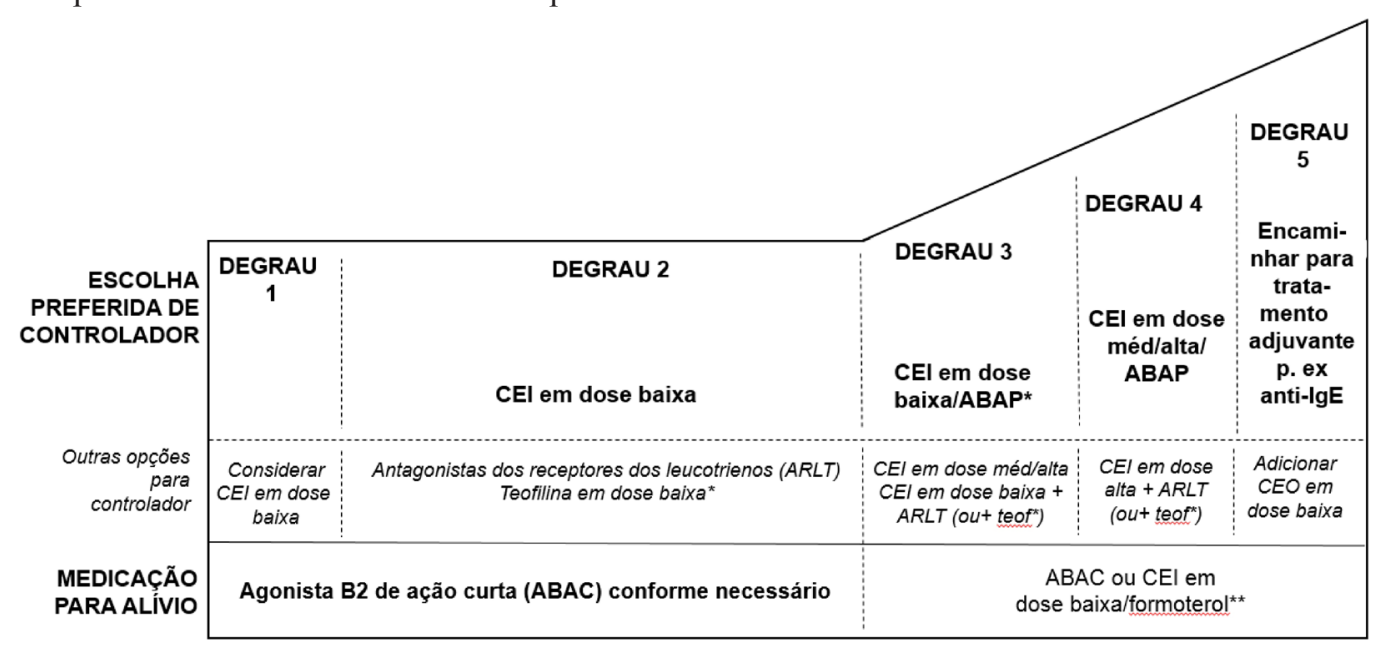

Legenda: CEI: corticosteroides inaláveis; ABAP: agonista beta 2 de ação prolongada; ARLT: antagonistas dos receptores dos leucotrienos; teof: teofilina; CEO: corticosteroides orais; ABAC: agonista de beta 2 de ação curta; *para crianças de 6-11 anos, a teofilina não é recomendada e o tratamento preferido do Degrau 3 é CEI em dose baixa; **CEI em dose baixa/formoterol é a medicação de alívio para doentes aos quais foi receitado budesonida em dose baixa/formoterol ou beclometasona em dose baixa/formoterol. Figura traduzida e adaptada de GINA 2015 (Goblal Initiative for Asthma ${ }^{1}$ ).

Figura 1. Abordagem medicamentosa para asma degrau a degrau 


\section{BREVE HISTÓRICO DOS EFEITOS DO EXERCÍCIO FÍSICO PARA ASMÁTICOS}

Apesar de todo o avanço no tratamento clínicomedicamentoso para pacientes asmáticos, muitas vezes os pacientes ainda permanecem com sintomas e nem sempre atingem o controle clínico adequado. Por causa disto, o Global Iniative for Asthma (GINA) sugere o uso de terapias não farmacológicas tais como o exercício físico, exercícios respiratórios e a cessação do tabagismo. Dentre estas técnicas nós destacamos o exercício físico, que é reconhecido atualmente como parte fundamental do programa de reabilitação para asmáticos ${ }^{8-10}$. Porém, até alguns anos atrás a indicação de exercício físico para pacientes asmáticos não era um consenso ${ }^{11}$, devido ao fato do exercício físico poder desencadear o broncoespasmo induzido pelo exercício (BIE). O BIE é uma condição transitória que ocasiona um estreitamento transitório das vias aéreas durante ou após o exercício físico e afeta, principalmente, os portadores de asma ${ }^{12}$. A prática do exercício intenso para pacientes asmáticos sempre foi também evitada porque a intensidade do BIE é tão mais intenso quanto maior a intensidade do exercício ${ }^{13}$.

Apesar do avanço nas pesquisas nos últimos anos, a recomendação de exercício físico para indivíduos com asma ainda se encontra limitada pelos profissionais da área da saúde não familiarizados com o tema devido ao temor de ocorrência do BIE. Esta relutância pela prescrição do exercício físico se devia ao fato de que, até início dos anos
2000, existiam poucos estudos científicos bem elaborados com objetivo de elucidar os benefícios do exercício físico para asmáticos com alguns viés metodológicos ${ }^{14,15}$; que incluíam pacientes com outras doenças pulmonares ${ }^{16}$; reduzido número de sujeitos ${ }^{17-19}$; e programas sem a descrição do nível de intensidade do esforço físico ${ }^{20}$. Em 2000, Ram et al. ${ }^{76}$ publicaram a primeira revisão sistemática que foi responsável pelo início da quebra do paradigma referente à prática de exercício físico para asmáticos, tanto em crianças ${ }^{15,17,21-23}$ como em adultos ${ }^{24-28}$. Mesmo com algumas limitações, os estudos incluídos nesta revisão demonstraram que os principais efeitos benéficos promovidos pelo exercício físico eram a melhora do condicionamento físico e da percepção de falta de ar (dispneia) $^{15,17,23-26,29,30}$.

Entretanto, a partir de 2000, surgiram diversos estudos com maior rigor metodológico ${ }^{13,31}$, demonstrando que o exercício físico pode reduzir o $\mathrm{BIE}^{32-35}$, a responsividade brônquica ${ }^{9,36,37}$, o uso de medicação corticosteroide $^{32}$ bem como melhorar os fatores de saúde relacionados à qualidade de vida ${ }^{10,32,38-40}$, a capacidade física anaeróbia ${ }^{18}$ e o controle clínico ${ }^{9,10,38,41,42}$. A partir de 2007, começaram a surgir também os estudos mostrando os efeitos do exercício físico na redução dos mecanismos envolvidos na manutenção da inflamação pulmonar ${ }^{43}$ tais como menor produção de imunoglobulinas alérgicas (IgG e $\operatorname{IgE}$ ) (38) e diminuição do estresse oxidativo ${ }^{44}$, da produção do óxido nítrico exalado ${ }^{10,44-46}$ e da migração de eosinófilos e linfócitos para as vias aéreas ${ }^{42,45}$ (Tabelas 1a e 1b).

Tabela 1a. Evolução do controle clínico pelos estudos em crianças asmática

\begin{tabular}{|c|c|c|c|}
\hline Autor/Ano & $\begin{array}{l}\text { Modalidade ou tipo de } \\
\text { exercício empregado }\end{array}$ & $\begin{array}{l}\text { Avaliação do controle } \\
\text { clínico }\end{array}$ & Principais mudanças obtidas após o EF regular \\
\hline Fitch et al., $1976^{22}$ & Natação & $\begin{array}{l}\text { Monitorização do escore de } \\
\text { sintomas e crises }\end{array}$ & Melhora da capacidade aeróbia e fatores psicológicos \\
\hline Huang et al., $1989^{23}$ & Natação & $\begin{array}{l}\text { Monitorização do escore de } \\
\text { sintomas }\end{array}$ & $\begin{array}{l}\text { Redução do absenteísmo escolar, visitas de sala de } \\
\text { emergência, internações, sibilância necessitando } \\
\text { menor quantidade de medicamentos diários }\end{array}$ \\
\hline King et al., $1989^{29}$ & $\begin{array}{l}\text { Alongamentos, calistênicos, } \\
\text { respiratórios, intervalados, jogos } \\
\text { esportivos adaptados e natação }\end{array}$ & Não investigado & Melhora da capacidade aeróbia \\
\hline Matsumoto et al., $1999^{30}$ & Natação & Não investigado & Melhora da capacidade aeróbia \\
\hline Neder et al., $1999^{15}$ & Calistênicos e aeróbios & $\begin{array}{l}\text { Monitorização do escore de } \\
\text { sintomas }\end{array}$ & $\begin{array}{l}\text { Melhora da capacidade aeróbia e redução da } \\
\text { medicação }\end{array}$ \\
\hline $\begin{array}{l}\text { van Veldhoven et al., } \\
2001^{20}\end{array}$ & $\begin{array}{l}\text { Circuito de exercícios aeróbios } \\
\text { e resistidos }\end{array}$ & Não investigado & Melhora da capacidade aeróbia e da resistência física \\
\hline Counil et al., 2003(18) & Aeróbios & Não investigado & Melhora das capacidades aeróbia e anaeróbia \\
\hline Weisgerber et al., $2003^{19}$ & Natação & $\begin{array}{l}\text { Monitorização do escore de } \\
\text { sintomas }\end{array}$ & Melhora da capacidade aeróbia \\
\hline Basaran et al., $2006^{39}$ & Basquete e respiratórios & $\begin{array}{l}\text { Monitorização do escore de } \\
\text { sintomas }\end{array}$ & Melhora da capacidade aeróbia, FSRQV e PFE \\
\hline Faneli et al., $2007^{32}$ & $\begin{array}{l}\text { Circuito de exercícios aeróbios } \\
\text { e resistidos }\end{array}$ & $\begin{array}{l}\text { Monitorização do escore de } \\
\text { sintomas }\end{array}$ & $\begin{array}{l}\text { Melhora da capacidade aeróbia, FSRQV, redução do } \\
\text { BIE e do uso de medicação }\end{array}$ \\
\hline Sidiropoulou et al., $2007^{33}$ & Futebol, treinamento intervalado & Não investigado & Melhora da capacidade aeróbia e $\mathrm{VEF}_{1}$ \\
\hline Moreira et al., $2008^{38}$ & $\begin{array}{l}\text { Alongamentos, calistênicos e } \\
\text { aeróbios }\end{array}$ & Não investigado & Redução de IgE \\
\hline
\end{tabular}

Legenda: EF=Exercício Físico; FSRQV=Fatores de Saúde Relacionados a Qualidade de Vida; $\mathrm{PFE}=$ Pico de Fluxo Expiratório; BIE= Broncoespasmo Induzido pelo Exercício; ACQ=Asthma Control Questionnaire; $\mathrm{AQLQ}=\mathrm{Asthma}$ Quality of Life Questionnaire; IgE=Imunoglobulina-E; $\mathrm{VEF}_{1}=$ Volume Expiratório Forçado no Primeiro Segundo 
Freitas PD, et al. Efeitos do exercício físico no controle clínico da asma.

Tabela 1b. Evolução na investigação do controle clínico pelos estudos em adultos asmáticos

\begin{tabular}{|c|c|c|c|}
\hline Autor/Ano & $\begin{array}{l}\text { Modalidade ou tipo de } \\
\text { exercício empregado }\end{array}$ & $\begin{array}{l}\text { Avaliação do controle } \\
\text { clínico }\end{array}$ & Principais mudanças obtidas após o EF regular \\
\hline $\begin{array}{l}\text { Fitch; Morton, } \\
1971^{25}\end{array}$ & $\begin{array}{l}\text { Corrida, ciclo ergômetro e } \\
\text { natação }\end{array}$ & Não investigado & Melhora da capacidade aeróbia e do $\mathrm{VEF}_{1}$ \\
\hline $\begin{array}{l}\text { Bundgaard et al., } \\
1982^{26}\end{array}$ & $\begin{array}{l}\text { Treino intervalado e } \\
\text { ginástica }\end{array}$ & Não investigado & Melhora da capacidade aeróbia \\
\hline $\begin{array}{l}\text { Freeman; Willians, } \\
1989^{27}\end{array}$ & Corrida & Não investigado & Melhora da capacidade aeróbia \\
\hline $\begin{array}{l}\text { Cochrane; Clark, } \\
1990^{28}\end{array}$ & $\begin{array}{l}\text { Calistênicos, resistidos, } \\
\text { ciclismo e corrida }\end{array}$ & $\begin{array}{l}\text { Monitorização do escore } \\
\text { de sintomas }\end{array}$ & $\begin{array}{l}\text { Melhora da capacidade aeróbia, anaeróbia e do } \\
V F_{1}\end{array}$ \\
\hline $\begin{array}{l}\text { Ahmaidi et al., } \\
1993^{71}\end{array}$ & Corrida & Não investigado & Melhora da capacidade aeróbia \\
\hline Varray et al., $1995^{69}$ & Natação & Não investigado & Melhora das capacidade aeróbia e anaeróbia \\
\hline $\begin{array}{l}\text { Gonçalves et al., } \\
2008^{10}\end{array}$ & Caminhada e respiratórios & $\begin{array}{l}\text { Avaliação e } \\
\text { monitorização dos } \\
\text { sintomas (AQLQ) }\end{array}$ & $\begin{array}{l}\text { Melhora das capacidade aeróbia, dos FSRQV, } \\
\text { redução dos sintomas e do óxido nítrico exalado }\end{array}$ \\
\hline Vempati et al., $2009^{34}$ & Ioga & Não investigado & $\begin{array}{l}\text { Melhora do } \mathrm{VEF}_{1} \text {, PFE e dos FSRQV, redução do } \\
\text { BIE }\end{array}$ \\
\hline Mendes et al., $2010^{41}$ & Respiratórios e aeróbios & $\begin{array}{l}\text { Monitorização do escore } \\
\text { de sintomas }\end{array}$ & $\begin{array}{l}\text { Melhora da capacidade aeróbia, dos FSRQV, } \\
\text { redução dos sintomas, ansiedade e depressão }\end{array}$ \\
\hline Mendes et al., $2011^{45}$ & Respiratórios e aeróbios & $\begin{array}{l}\text { Monitorização do escore } \\
\text { de sintomas }\end{array}$ & $\begin{array}{l}\text { Melhora da capacidade aeróbia, dos FSRQV, } \\
\text { redução dos sintomas e da inflamação pulmonar e } \\
\text { do óxido nítrico exalado }\end{array}$ \\
\hline Onur et al., $2011^{44}$ & Aeróbios & Não investigado & Redução do estresse oxidativo pulmonar \\
\hline Dogra et al., $2010^{47}$ & Aeróbios & $\begin{array}{l}\text { Avaliado pelo instrumento } \\
\text { ACQ }\end{array}$ & $\begin{array}{l}\text { Melhora da capacidade aeróbia, redução de } \\
\text { sintomas e melhora do }\end{array}$ \\
\hline Turner et al., $2010^{70}$ & Aeróbios e resistidos & $\begin{array}{l}\text { Monitorização do escore } \\
\text { de sintomas }\end{array}$ & Melhora da capacidade aeróbia e dos FSRQV \\
\hline Boyd et al., $2012^{63}$ & Aeróbios & $\begin{array}{l}\text { Avaliado pelo instrumento } \\
\text { ACQ }\end{array}$ & $\begin{array}{l}\text { Melhora da capacidade aeróbia, redução de } \\
\text { sintomas e melhora do controle clínico }\end{array}$ \\
\hline $\begin{array}{l}\text { Scichilone et al., } \\
2012^{36}\end{array}$ & Aeróbios & Não investigado & Redução da responsividade das vias aéreas \\
\hline Scott et al., $2012^{42}$ & Aeróbios e resistidos & $\begin{array}{l}\text { Avaliado pelo instrumento } \\
\text { ACQ }\end{array}$ & $\begin{array}{l}\text { Melhora da capacidade aeróbia, dos FSRQV, } \\
\text { redução do IMC, dos sintomas e da inflamação } \\
\text { pulmonar }\end{array}$ \\
\hline Andrade et al., $2014^{40}$ & Aeróbios & $\begin{array}{l}\text { Avaliação e monitorização } \\
\text { dos sintomas (AQLQ) }\end{array}$ & $\begin{array}{l}\text { Melhora da capacidade aeróbia, da pressão } \\
\text { máxima inspiratória, expiratória e dos FSRQV }\end{array}$ \\
\hline Scott et al., $2015^{46}$ & Aeróbios & $\begin{array}{l}\text { Avaliado pelo instrumento } \\
\text { ACQ }\end{array}$ & $\begin{array}{l}\text { Redução do óxido nítrico exalado e aumento da } \\
\text { expressão dos mediadores IL-6 e IL-1ra }\end{array}$ \\
\hline $\begin{array}{l}\text { França-Pinto et al., } \\
2015^{9}\end{array}$ & Respiratórios e aeróbios & $\begin{array}{l}\text { Avaliado pelo instrumento } \\
\text { ACQ e monitorização do } \\
\text { escore de sintomas }\end{array}$ & $\begin{array}{l}\text { Melhora da capacidade aeróbia, dos FSRQV, } \\
\text { redução dos sintomas, exacerbações, da } \\
\text { responsividade das vias aéreas, dos níveis de } \\
\text { ansiedade e depressão e da expressão de MCP-1 } \\
\text { e IL-6 }\end{array}$ \\
\hline
\end{tabular}

Legenda: $\mathrm{EF}=$ Exercício físico; $\mathrm{VEF} 1=$ Volume Expiratório Forçado no Primeiro Segundo; $\mathrm{ACQ}=\mathrm{Asthma}$ Control Questionnaire; $\mathrm{AQLQ}=\mathrm{Asthma}$ Quality of Life Questionnaire; FSRQV=Fatores de Saúde Relacionados a Qualidade de Vida; PFE= Pico de Fluxo Expiratório; IMC=Índice de Massa Corporea; MCP-1=Monocyte chemoattractant protein-1; IL=Interleucina 
Da mesma forma, avanços importantes foram mostrados quanto à melhora do controle clínico da asma utilizando instrumentos padronizados como os questionários como o ACQ e o $\mathrm{ACT}^{9,10,32,41,47}$. Além disto, estudos mostraram que o exercício físico é eficiente na redução dos sintomas ${ }^{9,10,41,42,45}$ e do número de exacerbações ${ }^{9,10,32,41}$, bem como na melhora dos valores de pico de fluxo expiratório ${ }^{9,10,41}$. Apesar do grande avanço e da relevância desses estudos mostrando todos os benefícios decorrentes da melhora do condicionamento físico ainda se desconhecem até o momento, os mecanismos antiinflamatórios indutores envolvidos na melhora do controle clínico nos pacientes asmáticos, o que parece a nova fronteira a ser rompida pelos futuros estudo ${ }^{43,48}$.

\section{Possíveis mecanismos anti-inflamatórios}

O conhecimento que temos até o presente momento, são oriundos de estudos experimentais com modelos de asma em animais que têm sido realizados com o objetivo de mimetizar os mecanismos que ocorrem nos pacientes expostos ao exercício físico ( ${ }^{49-52}$ ) e seus achados tem contribuído para elucidação de possíveis vias anti-inflamatórias ${ }^{53}$. Os efeitos descritos nestes modelos experimentais reforçam a redução da inflamação pulmonar ${ }^{49-52,54-57}$ e adicionam informações de que o exercício físico pode reverter o remodelamento das vias áreas e reduzir a resposta imune alérgica $^{52,54,55,58}$. Até o presente momento, pode-se destacar 3 possíveis vias anti-inflamatórias desencadeadas pelo aumento do condicionamento físico: i) aumento da expressão de mediadores anti-inflamatórios como IL-10 e IL-1 ra ${ }^{51,52,55}$, os quais tem papel na redução da resposta alérgica crônica ${ }^{59,60}$; ii) potencialização do glicocorticoide endógeno como imunossupressor natural ${ }^{61}$, devido aumento do seu receptor especifico nas células peribrônquicas e no músculo liso das vias aéreas ${ }^{50,51}$; e, iii) aumento da expressão do fator de transcrição nuclear FOXP3 por células dendríticas e redução da resposta alérgica imune $T h 2^{58}$. Apesar da relevância desses achados, é importante salientar que eles foram investigados em modelos animais de asma, não podendo, portanto, serem transpostos para a prática clínica. Nesse sentido, destaca-se a necessidade de futuras pesquisas com o objetivo de compreender os mecanismos indutores da redução da inflamação pulmonar induzida pelo exercício físico em pacientes asmáticos.

\section{PROGRAMA DE TREINAMENTO FÍSICO PARA ASMÁticos}

O programa de treinamento físico é recomendado para asmáticos com o objetivo de melhorar seu condicionamento físico ${ }^{62}$, coordenação neuromuscular e auto-confiança ${ }^{13}, \mathrm{e}$, consequentemente, levar a uma melhora clínica e funcional do paciente ${ }^{9,63,64}$. Porém, o tipo de treinamento, bem como sua intensidade, duração e frequência precisam ser considerados na organização do protocolo afim de promover as adaptações fisiológicas necessárias ${ }^{65}$ (Tabela 2).

Tabela 2. Exemplo de um protocolo de exercícios para asmáticos

\begin{tabular}{|c|c|c|}
\hline Etapas & Composição da sessão: opções de exercícios físicos & $\begin{array}{l}\text { Duração } \\
\text { (minutos) }\end{array}$ \\
\hline Avaliação inicial & $\begin{array}{l}\text { - Avaliar o PFE, a PA, a percepção subjetiva de esforço (Escala de Borg) e a FC. } \\
\text { - Uitlizar BD se o PFE for menor que } 70 \% \text { do melhor valor de PFE do paciente. }\end{array}$ & \multirow[b]{2}{*}{5 a 10} \\
\hline Aquecimento & $\begin{array}{l}\text { - Exercícios opcionais: alongamentos (MMSS e MMII). } \\
\text { - Tipos de exercício para aquecimento: caminhada, ciclismo ou jogos recreativos. } \\
\text { - Intensidade: } 50 \% \text { do } \mathrm{VO}_{2} \text { pico ou da FCmáx (leve). } \\
\text { - Equipamentos e recursos: bicicleta, esteira ergométrica, aéreas externas. } \\
\text { - Importância do aquecimento: evitar o desencadeamento do BIE. }\end{array}$ & \\
\hline $\begin{array}{l}\text { Condicionamento } \\
\text { físico }\end{array}$ & $\begin{array}{l}\text { - Tipos de exercícios aeróbios: caminhada, corrida, ciclismo ou natação. } \\
\text { - Exercício opcional: associar treino resistido. } \\
\text { - Intensidade: } 60 \text { a } 80 \% \text { do } \mathrm{VO}_{2} \text { pico ou da FCmax. } \\
\text { - Frequência: } 2 \text { a } 3 \text { vezes por semana. } \\
\text { - Monitorização: FC, } \mathrm{SpO}_{2} \text {, percepção subjetiva de esforço. } \\
\text { - Equipamentos e recursos: bicicleta, esteira ergométrica, piscina, execução de } \\
\text { exercícios em área livre, frequencímetro, oxímetro, percepção subjetiva de esforço } \\
\text { (Escala de Borg). }\end{array}$ & 20 a 30 \\
\hline Desquecimento & $\begin{array}{l}\text { - Exercício opcional: alongamentos (MMSS e MMII). } \\
\text { - Tipos de exercícios para voltar a calma: caminhada, ciclismo. } \\
\text { - Intensidade: } 50 \% \text { do } \mathrm{VO}_{2} \text { máx ou da FCmax (Leve). }\end{array}$ & \multirow{2}{*}{5 a 10} \\
\hline Avaliação final & $\begin{array}{l}\text { - Avaliar o PFE, PA, Escala de Borg e FC. } \\
\text { - Cuidado: deve-se tomar cuidado para saber que o PFE não apresenta uma queda } \geq 10 \% \\
\text { do valor obtido na avaliação inicial. }\end{array}$ & \\
\hline
\end{tabular}

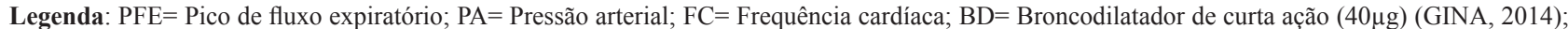
MMSS = Membros superiores; MMII = Membros inferiores; $\mathrm{VO}_{2}$ pico= Consumo pico de oxigênio; FCmáx = Frequência cardíaca máxima predita para a idade (ACSM, 2009); $\mathrm{SpO}_{2}=$ Saturação periférica de oxigênio; $\mathrm{BIE}=$ broncoespasmo induzido pelo exercício. 
Tipos de treinamento físico: baseado nas evidências da literatura até o momento, a maioria dos protocolos de treinamento físico para asmáticos incluem exercícios aeróbios tais como caminhada, corrida, ciclismo e natação combinados ou não com exercícios resistidos e alongamentos $^{66}$. Portanto, é importante ressaltar que a melhor modalidade de exercício é aquela em que o paciente melhor se adapte, tenha prazer em realizá-la, o local seja isento de fatores desencadeantes alérgicos e que respeite as condições econômicas e culturais do paciente. Assim, os pacientes terão maior aderência e rendimento na prática de exercícios ${ }^{67}$. Além disso, ressaltamos que estudos que mostraram melhora do controle clínico da asma utilizaram predominantemente treinos aeróbios ${ }^{9,63}$.

Duração da sessão: Os protocolos de treinamento físico para asmáticos variam muito quanto à duração de cada sessão. Porém, os estudos inclusos na última revisão sistemática $^{13}$ são realizados dentre os 30 e 90 minutos, duração que também é recomendado pelo consenso do Colégio Americano de Medicina do Esporte (ACSM) ${ }^{65}$. Importante ressaltar que essa duração de 30 a 90 minutos de exercícios geralmente é dividida em 3 etapas: aquecimento (5-15 min), condicionamento (20-60 min) e desaquecimento (5-15 min), que são importantes para evitar o desencadeamento do $\mathrm{BIE}^{68}$.

Intensidade: há uma grande variedade nos protocolos de treinamento para exercício para pacientes asmáticos e nem sempre a metodologia dos programas de treinamento físico em asmáticos são descritos adequadamente ${ }^{13}$ porém, a maioria dos estudos descreve uma intensidade que varia entre moderada a alta ${ }^{10,17,69}$ parametrizado, principalmente, pelo teste cardiopulmonar de esforço para determinar a potência aeróbia do paciente $\left(\mathrm{VO}_{2}\right.$ pico). Este teste é considerado padrão-ouro para prescrição da intensidade de exercício que é determinada através dos limiares ventilatórios e parametrizado durante o treinamento pela frequência cardíaca ${ }^{65}$. Porém, a prescrição do exercício utilizando este teste é limitada na prática clínica devido seu elevado custo e pela dificuldade de realizá-lo no dia-a-dia. Por isso, tem sido cada vez mais utilizada outras ferramentas tais como o teste de caminha de seis minutos ${ }^{70}$, o incremental Shuttle Walking Test ${ }^{71}$, a frequência cardíaca máxima predita para a idade ${ }^{14}$, a frequência cardíaca de reserva ${ }^{72}$, e até mesmo o limiar de lactato ${ }^{30}$.

Frequência: a grande parte dos estudos avaliando o efeito da reabilitação em pacientes asmáticos apontam que a frequência de 2 a 3 vezes por semana é suficiente para trazer benefícios aos asmáticos ${ }^{10,45,71}$. Apesar disso, é importante ressaltar que a frequência do programa de treinamento físico irá depender muito da intensidade e duração do treinamento, e que uma maior frequência poderá promover uma melhora da capacidade física mais rápida $\mathrm{e}$ de maior amplitude.
Duração do programa: a maioria dos protocolos de treinamento físico para pacientes asmáticos variam de 5 a 18 semanas, porém, a duração ideal deve ser aquela que produz os efeitos máximos desejados tomando-se o cuidado para não se tornar onerosa para o paciente ${ }^{73}$. Os estudos mais recentes têm mostrado que programas com 12 semanas de duração tem sido suficiente para melhorar o condicionamento físico com efeitos na melhora do controle clínico ${ }^{9,10}$.

\section{Programa de treinamento físico para asmáticos obesos}

A associação da asma com a obesidade leva a um fenótipo da doença que tem difícil controle e no qual os pacientes possuem mais sintomas e, portanto, devem receber um tratamento complementar para perda de peso associado à intervenção medicamentosa convencional ${ }^{1}$. Segundo recomendações da ACSM, os programas de perda de peso que combinem a redução do gasto calórico com aumento do nível de atividade física são os mais eficientes ${ }^{74}$. Portanto, o treinamento físico é indicado para pacientes asmáticos obesos não somente para a melhora de parâmetros relacionados ao condicionamento físico como também para potencializar a perda de peso.

Dessa forma, a prescrição de exercícios para pacientes asmáticos obesos deve ser adaptada para os objetivos específicos dessa população, levando em consideração as limitações causadas pela obesidade e o treinamento físico deve seguir algumas recomendações especiais tais como um aumento da duração e da frequência de exercícios, com diminuição da intensidade inicial e da progressão dos exercícios. Além disso, recomenda-se associar exercícios de resistência ao treino aeróbio afim de oferecer um programa de treinamento físico mais equilibrado de maneira a aumentar a massa magra e o consumo metabólico basal e também preparando o paciente para sustentar uma maior demanda de atividade aeróbia, além de diminuir o risco de lesões musculoesqueléticas e o estresse fisiológico em atividades diárias ${ }^{75}$. Importante ressaltar que tanto a avaliação inicial quanto a supervisão do treino devem ser mais criteriosos, já que a obesidade, geralmente, está acompanhada de algumas comorbidades importantes (como dislipidemia, hipertensão, hiperglicemia, dentre outros) que podem aumentar a classificação do risco para essa população. Dessa forma, recomenda-se monitorar não somente as medicações para o tratamento da asma, como também das outras comorbidades, priorizar o cicloergômetro ao realizar teste cardiopulmonar de esforço máximo, adaptar os exercícios, equipamentos sempre que possível, principalmente na presença de patologias ortopédicas e/ou musculoesqueléticas ${ }^{65}$ (Tabela 3). 
Freitas PD, et al. Efeitos do exercício físico no controle clínico da asma.

Tabela 3. Exemplo de um protocolo de exercícios para asmáticos obesos

\begin{tabular}{|c|c|c|}
\hline Etapas & Composição da sessão: opções de exercícios físicos & $\begin{array}{l}\text { Duração } \\
\text { (minutos) }\end{array}$ \\
\hline Avaliação inicial & $\begin{array}{l}\text { - Avaliar comorbidades e medicações em uso. } \\
\text { - Avaliar o PFE, PA, Escala de Borg e FC. } \\
\text { - Utilizar BD se o PFE for menor que } 70 \% \text { do melhor valor de PFE do paciente. }\end{array}$ & \\
\hline Aquecimento & $\begin{array}{l}\text { - Recomenda--se realizar alongamentos (MMSS e MMII). } \\
\text { - Tipos de exercício para aquecimento: caminhada, ciclismo ou jogos recreativos. } \\
\text { - Equipamentos e recursos: bicicleta, esteira ergométrica, aéreas externas. } \\
\text { - Intensidade: } 50 \% \text { do } \mathrm{VO}_{2} \text { máx ou da FCmáx (leve). } \\
\text { - Importância do aquecimento: evitar o desencadeamento do BIE. }\end{array}$ & 5 a 15 \\
\hline $\begin{array}{l}\text { Condicionamento } \\
\text { físico }\end{array}$ & $\begin{array}{l}\text { - Tipos de exercícios aeróbios: caminhada, corrida, ciclismo ou natação. } \\
\text { - Recomenda-se associar treino resistido ao aeróbio em forma de circuito. } \\
\text { - Intensidade: } 60 \text { a } 75 \% \text { do } \mathrm{VO}_{2} \text { máx ou da } \mathrm{FCmax} \text {. } \\
\text { - Frequência: } 2 \text { a } 3 \text { vezes/semana inicialmente com progressão para } 3 \text { a } 5 \text { x/semana. } \\
\text { - Monitorização: } \mathrm{FC}, \mathrm{SpO}_{2} \text {, percepção subjetiva de esforço. } \\
\text { - Equipamentos e recursos: bicicleta, esteira ergométrica, piscina, execução de exercícios } \\
\text { em área livre, frequencímetro, oxímetro, Escala de Borg. }\end{array}$ & 40 a 50 \\
\hline Desquecimento & $\begin{array}{l}\text { - Exercício opcional: alongamentos (MMSS e MMII). } \\
\text { - Tipos de exercícios para voltar a calma: caminhada, ciclismo. } \\
\text { - Intensidade: } 50 \% \text { do } \mathrm{VO}_{2} \text { máx ou da FCmáx (Leve). }\end{array}$ & 5 a 10 \\
\hline Avaliação final & - Avaliar o PFE, PA, Escala de Borg e FC. & \\
\hline
\end{tabular}

Legenda: $\mathrm{PFE}=$ Pico de fluxo expiratório; $\mathrm{PA}=$ Pressão arterial; $\mathrm{FC}=$ Frequência cardíaca; $\mathrm{BD}=$ Broncodilatador de curta ação; $\mathrm{MMSS}=\mathrm{Membros}$ superiores; $\mathrm{MMII}=$ Membros inferiores; $\mathrm{VO}_{2}$ pico= Consumo pico de oxigênio; $\mathrm{FCmáx}=$ Frequência cardíaca máxima predita para a idade; SpO $=$ Saturação periférica de oxigênio; BIE= broncoespasmo induzido pelo exercício.

\section{CONSIDERAÇÕES FINAIS}

Evidências científicas recentes têm reforçado a importância do treinamento físico no programa de reabilitação para pacientes asmáticos, uma vez que a melhora do condicionamento físico exerce um importante papel

\section{REFERÊNCIAS}

1. GINA - Global Initiative for Asthma. Global Strategy for Asthma Management and Prevention. National Institutes of Health. National Heart, Lung and Blood Institut of Health, Bethesda. Uptated 2015 [cited 2015 June]. Available from: http://www.ginasthma.org/.

2. GINA - Global Initiative for Ashtma. Global Burden of asthma. National Institutes of Health. National Heart, Lung and Blood Institut of Health, Bethesda. Uptated 2014 [cited 2015 June]. Available from: http://www.ginasthma.org/.

3. Leite M, Ponte EV, Petroni J, D’Oliveira Junior A, Pizzichini E, Cruz AA. Evaluation of the asthma control questionnaire validated for use in Brazil. J Bras Pneumol. 2008;34(10):75663. http://dx.doi.org/10.1590/S1806-37132008001000002.

4. Roxo JP, Ponte EV, Ramos DC, Pimentel L, D'Oliveira Junior A, Cruz AA. Portuguese-language version of the Asthma Control Test: validation from use of Brazil. J Bras Pneumol. 2010;36(2):159-66. http://dx.doi.org/10.1590/ S1806-37132010000200002. na melhora clínica e funcional desses pacientes, principalmente naqueles com diagnóstico moderado a grave da doença, sugerindo sua indicação na prática clínica para os pacientes que estão em tratamento clínico-medicamentoso.

5. Juniper EF, Svensson K, Mork AC, Stahl E. Measurement properties and interpretation of three shortened versions of the asthma control questionnaire. Respir Med. 2005;99(5):553-8.

6. Juniper EF, Bousquet J, Abetz L, Bateman ED, Committee G. Identifying 'well-controlled' and 'not well-controlled' asthma using the Asthma Control Questionnaire. Respir Med. 2006;100(4):616-21.

7. Schatz M, Sorkness CA, Li JT, Marcus P, Murray JJ, Nathan RA, et al. Asthma Control Test: reliability, validity, and responsiveness in patients not previously followed by asthma specialists. J Allergy Clin Immunol. 2006;117(3):549-56.

8. Meyer A, Gunther S, Volmer T, Taube K, Baumann HJ. A 12-month, moderate-intensity exercise training program improves fitness and quality of life in adults with asthma: a controlled trial. BMC Pulmonary Med. 2015;15:56.

9. Franca-Pinto A, Mendes FA, de Carvalho-Pinto RM, Agondi RC, Cukier A, Stelmach R, et al. Aerobic training decreases bronchial hyperresponsiveness and systemic inflammation 
Freitas PD, et al. Efeitos do exercício físico no controle clínico da asma.

in patients with moderate or severe asthma: a randomised controlled trial. Thorax. 2015;70(8):732-9.

10. Gonçalves RC, Nunes, M.P.T., Cukier, A., Stelmach, R., Martins, M.A., Carvalho. C.R.F. Effects of an aerobic physical training program on psychosocial characteristics, quality-oflife, symptoms and exhaled nitric oxide in individuals with moderate or severe persistent asthma. Rev Bras Fisioter. 2008;12(2):9.

11. Disabella V, Sherman C. Exercise for asthma patients: little risk, big rewards. Phys Sports Med. 1998;26(6):75-84.

12. Teixeira RN, Gonçalves RC, Costa LAR, Silva LBdBe, Martins MdA, Teixeira LR, et al. Efeito do tratamento clínico de um corredor de longa distância com broncoespasmo induzido pelo exercício: relato de caso. Rev Bras Med Esporte. 2009; $15: 132-4$.

13. Carson KV, Chandratilleke MG, Picot J, Brinn MP, Esterman AJ, Smith BJ. Physical training for asthma. Cochrane Database Syst Rev. 2013;9:CD001116.

14. Emtner M, Herala M, Stalenheim G. High-intensity physical training in adults with asthma. A 10-week rehabilitation program. Chest. 1996;109(2):323-30.

15. Neder JA, Nery LE, Silva AC, Cabral AL, Fernandes AL. Short-term effects of aerobic training in the clinical management of moderate to severe asthma in children. Thorax. 1999;54(3):202-6.

16. Cambach W, Chadwick-Straver RV, Wagenaar RC, van Keimpema AR, Kemper HC. The effects of a communitybased pulmonary rehabilitation programme on exercise tolerance and quality of life: a randomized controlled trial. Eur Respir J. 1997;10(1):104-13.

17. Varray AL, Mercier JG, Terral CM, Prefaut CG. Individualized aerobic and high intensity training for asthmatic children in an exercise readaptation program. Is training always helpful for better adaptation to exercise? Chest. 1991;99(3):579-86.

18. Counil FP, Varray A, Matecki S, Beurey A, Marchal P, Voisin $\mathrm{M}$, et al. Training of aerobic and anaerobic fitness in children with asthma. J Pediatrics. 2003;142(2):179-84.

19. Weisgerber MC, Guill M, Weisgerber JM, Butler H. Benefits of swimming in asthma: effect of a session of swimming lessons on symptoms and PFTs with review of the literature. J Asthma. 2003;40(5):453-64.

20. van Veldhoven NH, Vermeer A, Bogaard JM, Hessels MG, Wijnroks L, Colland VT, et al. Children with asthma and physical exercise: effects of an exercise programme. Clin Rehabil. 2001;15(4):360-70.

21. Sly RM, Harper RT, Rosselot I. The effect of physical conditioning upon asthmatic children. Ann Allergy. 1972;30(2):86-94.

22. Fitch KD, Morton AR, Blanksby BA. Effects of swimming training on children with asthma. Arch Dis Childhood. 1976;51(3):190-4.

23. Huang SW, Veiga R, Sila U, Reed E, Hines S. The effect of swimming in asthmatic children--participants in a swimming program in the city of Baltimore. J Asthma. 1989;26(2):11721.

24. Clark CJ, Cochrane LM. Physical activity and asthma. Curr
Opin Pulmonary Med. 1999;5(1):68-75.

25. Fitch KD, Morton AR. Specificity of exercise in exerciseinduced asthma. Br Med J. 1971;4(5787):577-81.

26. Bundgaard A, Ingemann-Hansen T, Halkjaer-Kristensen J, Schmidt A, Bloch I, Andersen PK. Short-term physical training in bronchial asthma. Br J Dis Chest. 1983;77(2):14752.

27. Freeman W, Nute MG, Williams C. The effect of endurance running training on asthmatic adults. Br J Sports Med. 1989;23(2):115-22.

28. Cochrane LM, Clark CJ. Benefits and problems of a physical training programme for asthmatic patients. Thorax. 1990;45(5):345-51.

29. King JM, Noakes TD, Weinberg EG. Physiological Effects of a Physical Training Program in Children With ExerciseInduced Asthma. Pediatric Exerc Sci. 1989;1:137-44.

30. Matsumoto I, Araki H, Tsuda K, Odajima H, Nishima S, Higaki $\mathrm{Y}$, et al. Effects of swimming training on aerobic capacity and exercise induced bronchoconstriction in children with bronchial asthma. Thorax. 1999;54(3):196-201.

31. Ram FS, Robinson SM, Black PN, Picot J. Physical training for asthma. Cochrane Database Syst Rev. 2005(4):CD001116.

32. Fanelli A, Cabral AL, Neder JA, Martins MA, Carvalho CR. Exercise training on disease control and quality of life in asthmatic children. Med Sci Sports Exerc. 2007;39(9):147480.

33. Sidiropoulou MP, Fotiadou EG, Tsimaras VK, Zakas AP, Angelopoulou NA. The effect of interval training in children with exercise-induced asthma competing in soccer. J Strength Conditioning Res. 2007;21(2):446-50.

34. Vempati R, Bijlani RL, Deepak KK. The efficacy of a comprehensive lifestyle modification programme based on yoga in the management of bronchial asthma: a randomized controlled trial. BMC Pulmonary Med. 2009;9:37.

35. El-Akkary IM, Abdel-Fatah Zel K, El-Seweify Mel S, ElBatouti GA, Aziz EA, Adam AI. Role of leukotrienes in exercise-induced bronchoconstriction before and after a pilot rehabilitation training program. Int J General Med. 2013;6:631-6.

36. Scichilone N, Morici G, Zangla D, Arrigo R, Cardillo I, Bellia V, et al. Effects of exercise training on airway closure in asthmatics. J Applied Physiol. 2012;113(5):714-8.

37. Wicher IB, Ribeiro MA, Marmo DB, Santos CI, Toro AA, Mendes RT, et al. Effects of swimming on spirometric parameters and bronchial hyperresponsiveness in children and adolescents with moderate persistent atopic asthma. J Pediatria. 2010;86(5):384-90.

38. Moreira A, Delgado L, Haahtela T, Fonseca J, Moreira $\mathrm{P}$, Lopes $\mathrm{C}$, et al. Physical training does not increase allergic inflammation in asthmatic children. Eur Respir J. 2008;32(6):1570-5.

39. Basaran S, Guler-Uysal F, Ergen N, Seydaoglu G, BingolKarakoc G, Ufuk Altintas D. Effects of physical exercise on quality of life, exercise capacity and pulmonary function in children with asthma. J Rehabil Med. 2006;38(2):130-5.

40. Andrade LB, Britto MC, Lucena-Silva N, Gomes RG, 
Freitas PD, et al. Efeitos do exercício físico no controle clínico da asma.

Figueroa JN. The efficacy of aerobic training in improving the inflammatory component of asthmatic children. Randomized trial. Respir Med. 2014;108(10):1438-45.

41. Mendes FA, Goncalves RC, Nunes MP, Saraiva-Romanholo BM, Cukier A, Stelmach R, et al. Effects of aerobic training on psychosocial morbidity and symptoms in patients with asthma: a randomized clinical trial. Chest. 2010;138(2):331-7.

42. Scott HA, Gibson PG, Garg ML, Pretto JJ, Morgan PJ, Callister R, et al. Dietary restriction and exercise improve airway inflammation and clinical outcomes in overweight and obese asthma: a randomized trial. Clin Exp Allergy. 2013;43(1):36-49.

43. Pakhale S, Luks V, Burkett A, Turner L. Effect of physical training on airway inflammation in bronchial asthma: a systematic review. BMC Pulmonary Med. 2013;13:38.

44. Onur E, Kabaroglu C, Gunay O, Var A, Yilmaz O, Dundar P, et al. The beneficial effects of physical exercise on antioxidant status in asthmatic children. Allergologia Immunopathol. 2011;39(2):90-5.

45. Mendes FA, Almeida FM, Cukier A, Stelmach R, Jacob-Filho W, Martins MA, et al. Effects of aerobic training on airway inflammation in asthmatic patients. Med Scie Sports Exerc. 2011;43(2):197-203.

46. Scott HA, Latham JR, Callister R, Pretto JJ, Baines K, Saltos $\mathrm{N}$, et al. Acute exercise is associated with reduced exhaled nitric oxide in physically inactive adults with asthma. Ann Allergy Asthma Immunol. 2015;114(6):470-9.

47. Dogra S. Physical training is well tolerated, leads to improvements in cardiopulmonary fitness and is not associated with adverse outcomes in people with asthma. Evidence Based Nursing. 2013;16(1):20-1.

48. Eichenberger PA, Diener SN, Kofmehl R, Spengler CM. Effects of exercise training on airway hyperreactivity in asthma: a systematic review and meta-analysis. Sports Med. 2013;43(11):1157-70.

49. Pastva A, Estell K, Schoeb TR, Atkinson TP, Schwiebert LM. Aerobic exercise attenuates airway inflammatory responses in a mouse model of atopic asthma. J Immunol. 2004;172(7):4520-6.

50. Pastva A, Estell K, Schoeb TR, Schwiebert LM. RU486 blocks the anti-inflammatory effects of exercise in a murine model of allergen-induced pulmonary inflammation. Brain Behav Immun. 2005;19(5):413-22.

51. Silva RA, Almeida FM, Olivo CR, Saraiva-Romanholo BM, Martins MA, Carvalho CR. Exercise reverses OVA-induced inhibition of glucocorticoid receptor and increases antiinflammatory cytokines in asthma. Scand J Med Sci Sports. 2015.

52. Vieira RP, Claudino RC, Duarte AC, Santos AB, Perini A, Faria Neto HC, et al. Aerobic exercise decreases chronic allergic lung inflammation and airway remodeling in mice. American J Respir Crit Care Med. 2007;176(9):871-7.

53. Luks V, Burkett A, Turner L, Pakhale S. Effect of physical training on airway inflammation in animal models of asthma: a systematic review. BMC Pulmonary Med. 2013;13:24.

54. Silva RA, Almeida FM, Olivo CR, Saraiva-Romanholo BM, Martins MA, Carvalho CR. Airway remodeling is reversed by aerobic training in a murine model of chronic asthma. Scand J Med Scie Sports. 2015;25(3):e258-66.

55. Silva RA, Vieira RP, Duarte AC, Lopes FD, Perini A, Mauad $\mathrm{T}$, et al. Aerobic training reverses airway inflammation and remodelling in an asthma murine model. Eur Respir J. 2010;35(5):994-1002.

56. Vieira RP, Silva RA, Oliveira-Junior MC, Greiffo FR, Ligeiro-Oliveira AP, Martins MA, et al. Exercise deactivates leukocytes in asthma. Int J Sports Med. 2014;35(7):629-35.

57. Vieira RP, Toledo AC, Ferreira SC, Santos AB, Medeiros MC, Hage M, et al. Airway epithelium mediates the antiinflammatory effects of exercise on asthma. Respir Physiol Neurobiol. 2011;175(3):383-9.

58. Lowder T, Dugger K, Deshane J, Estell K, Schwiebert LM. Repeated bouts of aerobic exercise enhance regulatory $\mathrm{T}$ cell responses in a murine asthma model. Brain Behav Immun. 2010;24(1):153-9.

59. Arend WP. The balance between IL-1 and IL-1Ra in disease. Cytokine Growth Factor Rev. 2002;13(4-5):323-40.

60. Hovsepian E, Penas F, Siffo S, Mirkin GA, Goren NB. IL-10 inhibits the NF-kappaB and ERK/MAPK-mediated production of pro-inflammatory mediators by up-regulation of SOCS-3 in Trypanosoma cruzi-infected cardiomyocytes. PloS One. 2013;8(11):e79445.

61. Barnes PJ. Anti-inflammatory actions of glucocorticoids: molecular mechanisms. Clin Sci. 1998;94(6):557-72.

62. Arandelovic M, Stankovic I, Nikolic M. Swimming and persons with mild persistant asthma. Scient World J. 2007;7:1182-8

63. Boyd A, Yang CT, Estell K, Ms CT, Gerald LB, Dransfield $\mathrm{M}$, et al. Feasibility of exercising adults with asthma: a randomized pilot study. Allergy Asthma Clin Immunol. 2012;8(1):13

64. Weisgerber M, Webber K, Meurer J, Danduran M, Berger $\mathrm{S}$, Flores $\mathrm{G}$. Moderate and vigorous exercise programs in children with asthma: safety, parental satisfaction, and asthma outcomes. Pediatric Pulmonol. 2008;43(12):1175-82.

65. American College of Sports Medicine (ACSM). ACSM's guidelines for exercise testing and prescription. 9th ed. Philadelphia: Lippincott Williams \& Wilkins; 2013.

66. Avallone K, McLeish A. Asthma and aerobic exercise: areview of empirical literature. J Ashtma. 2013;50(2):109-16. doi: 10.3109/02770903.2012.759963.

67. Felipe F, Stelmach R, Martins M, Carvalho C. Papel do exercício físico no tratamento do paciente asmático. Pneumologia Paulista. 2012;26(1).

68. Cawley J. Exercise-Induced Asthma. J Pharm Pract. 2003;16(1):59-67.

69. Varray AL, Mercier JG, Prefaut CG. Individualized training reduces excessive exercise hyperventilation in asthmatics. Int Zeitschrift fur Rehabilitationsforschung. Rev Int Recherches Readapt. 1995;18(4):297-312.

70. Turner S, Eastwood P, Cook A, Jenkins S. Improvements in symptoms and quality of life following exercise training in older adults with moderate/severe persistent asthma. Respir Int Rev Thorac Dis. 2011;81(4):302-10. 
Freitas PD, et al. Efeitos do exercício físico no controle clínico da asma.

71. Ahmaidi SB, Varray AL, Savy-Pacaux AM, Prefaut CG. Cardiorespiratory fitness evaluation by the shuttle test in asthmatic subjects during aerobic training. Chest. 1993;103(4):1135-41.

72. Vallet G, Ahmaidi S, Serres I, Fabre C, Bourgouin D, Desplan $\mathrm{J}$, et al. Comparison of two training programmes in chronic airway limitation patients: standardized versus individualized protocols. Eur Respir J. 1997;10(1):114-22.

73. Ries AL, Bauldoff GS, Carlin BW, Casaburi R, Emery CF, Mahler DA, et al. Pulmonary rehabilitation: joint ACCP/ AACVPR evidence-based clinical practice guidelines. Chest. 2007;131(5 Suppl):4S-42S.
74. Thompson PD, Arena R, Riebe D, Pescatello LS, American College of Sports M. ACSM's new preparticipation health screening recommendations from ACSM's guidelines for exercise testing and prescription, ninth edition. Curr Sports Med Rep. 2013;12(4):215-7.7

75. American College of Sports Medicine (ACSM). Exercise prescription for Other Clinical Populations. In: ACSM's Guidelines for Exercise Testing and Prescription. 8th ed. Philadelphia: Lippincott Williams \& Wilkins; 2009. p.266-72.

76. Ram FS, Robinson SM, Black PN. Effects of physical training in asthma: a systematic review. Br J Sports Med. 2000;34(3):162-7. 